\title{
La Universidad Nacional Autónoma de México: pionera en la sistematización de la información científica iberoamericana
}

\author{
The National Autonomous University of México: leader institution in the production \\ of the databases of Latin American information
}

\section{María Ángela Torres Verdugo (1) y Carmen García Colorado (2)}

(1) IRESIE, Centro de Estudios sobre la Universidad, Universidad Nacional Autónoma de México, Edificio de la Unidad Biobliográfica, principal, Ciudad Universitaria, 04510 México, D. F. (México), angelat@ servidor.unam.mx (2) Dirección General de Bibliotecas, Universidad Nacional Autónoma de México, Biblioteca Central, Entrepiso으, Circuito Interior, Ciudad Universitaria, 04510 México, D. F. (México)

\begin{abstract}
Resumen
Se presentan las características de las bases de datos generadas en los institutos y centros de la Universidad Nacional Autónoma de México (UNAM) cuyo contenido aborda el conocimiento científico publicado en revistas de los países de América Latina, el Caribe, España y Portugal sobre Ciencias Sociales y Humanidades. El estudio se realizó a partir de la revisión de las páginas Web de los institutos y centros y entrevistas telefónicas con los responsables de las bases de datos. Se describen antecedentes, características, dependencias productoras, cobertura temática y geográfica, servicios, formas de acceso a las bases de datos a través de Internet. En particular se examina el IRESIE, base de datos sobre educación iberoamericana; CLASE (Citas Latinoamericanas en Ciencias Sociales y Humanidades); PERIODICA, (Indice de Revistas Latinoamericanas en Ciencias), FILOS (Filosofía en México); INFOBILA (Información y Bibliotecología Latinoamericana); y LATINDEX, (Sistema regional de información en línea para revistas científicas de América Latina, el Caribe, España y Portugal); todas accesibles en forma gratuita.
\end{abstract}

Palabras clave: Bases de datos bibliográficas. Sistemas de información. Universidad Nacional Autónoma de México. América Latina. Iberoamérica.

\section{1. Introducción}

La UNAM es la institución de enseñanza superior más importante de México, la primera de América Latina y la número 59 en el ranking mundial.

Para dar una idea de la importancia social y de las dimensiones de la UNAM, mencionamos que la inauguración de la Ciudad Universitaria en 1957 constituyó la cristalización de aspiraciones de ascenso educativo y movilidad social, largamente acariciadas por amplios sectores de las

\begin{abstract}
The databases generated in the institutes and centers of the Universidad Nacional Autónoma de México (UNAM) are analyzed, where the scientific knowledge published in the countries of Latin America, Caribbean, Spanish and Portuguese journals on Humanities and Social Sciences is referred. Methodology. From the review of the web pages of the institutes that approach these disciplines, we identified some databases. We interview the persons in charge of the development of the databases to obtain information about the content, level of analysis, number of records, software, products and services. This paper describes, the characteristics, subject and geographic coverage, services and forms of access to these data bases. The databases analyzed are IRESIE, iberoamerican education database; CLASE (Latin American Citation Index in Social Sciences and Humanities) PERIODICA; (Index to Latin American Sciences Journals); FILOS (Mexican Philosophy); and INFOBILA (Latin American Library and Information Sciene); and LATINDEX (Regional system of online information for science journals of Latin America, Caribe, Spain and Portugal).
\end{abstract}

Keywords: Bibliographic databases. Information systems. National Autonomous University of Mexico. Latin America. Iberoamerica.

clases medias y bajas del país, por tratarse de una universidad pública y gratuita, ya que permitió el acceso a grandes sectores de la población antes excluidos de la educación superior. De ella, han egresado miles de profesionales que participan en la vida pública y que han fundado y fortalecido cientos de instituciones públicas y privadas del país.

En cuanto al tamaño de la población universitaria, de acuerdo con datos de la Agenda Estadística de 2007, la comunidad universitaria está integrada por 292,889 estudiantes $-21,230$ de 
posgrado, 163,368 de licenciatura, 106,298 de bachillerato, 1264 técnicos y 729 de la Escuela Nacional de Música- y 42,347 académicos -2, 337 investigadores, 5,417 profesores de carrera, 3,950 Técnicos académicos, 26,291 profesores de asignatura, 4,011 Ayudantes de profesor y 341 de otros tipos.

\section{Investigación}

La UNAM genera el 50 \% de la investigación que se produce en México. Las actividades de investigación fundamentalmente se desarrollan en los centros e institutos de investigación, que se agrupan en dos grandes subsistemas: Investigación Científica (SIC), e Investigación en Humanidades (SIH)

El Subsistema de la Investigación Científica incluye 19 institutos y 10 centros, agrupados en tres grandes áreas del conocimiento: Ciencias Químico-Biológicas y de la Salud, Ciencias Físico-Matemáticas y Ciencias de la Tierra e Ingenierías. Incluye también el Programa de Investigación Multidisciplinaria, Proyectos Universitarios de Liderazgo Académico, cinco Programas Universitarios de Ciencia, la Dirección General de Divulgación de la Ciencia, la Coordinación de Plataformas Oceanográficas y la Secretaría Ejecutiva de la Reserva Ecológica del Pedregal de San Ángel.

El Subsistema de Humanidades (SH) se compone de la Coordinación de Humanidades, 9 Institutos de Investigación: Antropológicas, Económicas, Bibliográficas, Estéticas, Filológicas, Históricas, Filosóficas, Jurídicas, Sociales y sobre la Universidad y la Educación, 7 Centros de Investigaciones: sobre América Latina y el Caribe, de Investigaciones Bibliotecológicas, Interdisciplinarias en Ciencias y Humanidades, Regional de Investigaciones Multidisciplinarias, sobre América del Norte, Centro Peninsular en Humanidades y Ciencias Sociales y el de Enseñanza en Lenguas Extranjeras. Los Programas de Estudios de Género y de Estudios sobre la Ciudad, además de la Unidad de Estudios Regionales: La Ciénaga.

\section{Recursos de información}

\subsection{Bibliotecas}

Para apoyar las actividades sustantivas de docencia, investigación y difusión, se cuenta con una infraestructura de información muy sólida, única en el país por su tamaño, calidad y antigüedad de sus colecciones: 142 bibliotecas, las cuales albergan $6,106,012$ volúmenes, que corresponden a 2,262,796 títulos de libros; de estos 7,175 están en formato electrónico; 3,288
477 fascículos de revistas que corresponden a 69,222 títulos; de ellas, 19,266 están en formato electrónico; 653,849 tesis, de las cuales 53,940 están digitalizadas.

El presupuesto en 2006 asignado a la compra de libros impresos y electrónicos fue de $6,478,979$ dólares y de 15,210,862 dólares para suscripción a revistas en papel y electrónicas incluyendo 230 bases de datos internacionales, que son consultadas principalmente por docentes, investigadores y alumnos de posgrado. En el campus universitario residen además la Biblioteca y la Hemeroteca Nacional.

\subsection{Bases de datos producidas por la UNAM}

En los países desarrollados, a partir de los sesentas se crearon sistemas de información y bases de datos, en los que cada disciplina imprimió características particulares, derivadas de sus necesidades propias, por ejemplo, Medline para el área médica y Eric para el área educativa.

Siguiendo esta corriente mundial, en 1971 se funda en la UNAM el otrora Centro de Información Científica y Humanística $(\mathrm{CICH})$, que abarcaba las dos grandes áreas del conocimiento: las ciencias exactas y experimentales y las ciencias sociales y las humanidades. Desde su inicio empezó a desarrollar productos de información, entre ellos: los servicios de búsquedas bibliográficas, alertas, recuperación de documentos, índice de citas de autores y se construyeron bases de datos referenciales.

En 1973, el CICH inicia la búsqueda, recopilación y sistematización de la producción científica de autores mexicanos y latinoamericanos del área científica y humanística que publicaban sus trabajos en revistas internacionales. A partir de esta selección se constituyó la "Bibliografía Mexicana" y la "Bibliografía Latinoamericana". (BIBLAT). Esta información se difundía a la comunidad académica a través de un boletín quincenal de ALERTA que sirvió de fuente para realizar estudios bibliométricos sobre la productividad científica de México y América Latina, que fueron presentados en el 38 Congreso Mundial de la Federación Internacional de Documentación (1976), con el tema Información y desarrollo, que abordó la dispersión de la información generada en América Latina y publicada en revistas de la región.

En este contexto se crean en el $\mathrm{CICH}$ las bases de datos CLASE Y PERIODICA en 1975 y 1978 respectivamente. Así mismo, en otras dependencias universitarias se fueron generando otras bases como IRESIE, FILOS, INFOBILA, 
BIVE, SIPAL, ALFA, GENEROS, GEOMEX, BEXART, MECS, MEXICOARTE, LATINARTE y PACIOLI, todas ellas con el fin recopilar, analizar y difundir la información de todos los campos del conocimiento generada en México y en la región iberoamericana.

Algunas de estas bases de datos han desaparecido a la fecha, debido a la falta de interés de algunos funcionarios $O$ a la limitación de recursos. Otras - como CLASE, PERIODICA, IRESIE, FILOS e INFOBILA - tienden a consolidarse, y han ido adecuando sus productos al avance tecnológico que facilita la transformación de las bases de datos referenciales, o con resúmenes en bases de datos de texto completo.

A continuación describimos las bases de datos cuya cobertura abarca artículos publicados en revistas de la región iberoamericana.

\subsubsection{CLASE y PERIODICA}

Como se ha señalado, estas bases nacen en 1975 y 1978 respectivamente en el Departamento de Bibliografía Latinoamericana del otrora $\mathrm{ClCH}$, ahora perteneciente a la Subdirección de Servicios Especializados de la Dirección General de Bibliotecas (DGB). En conjunto ofrecen el acceso a más de 550 mil registros bibliográficos de artículos, ensayos, reseñas de libros, revisiones bibliográficas, entrevistas, estadísticas y otros documentos, publicados en alrededor de 2700 títulos de revistas académicas editadas por diferentes instituciones de México, América Latina y el Caribe, especializadas en ciencias exactas, ingenierías, ciencias sociales y humanidades.

Cubren prácticamente todas las áreas del conocimiento que se estudian e investigan en la UNAM. CLASE engloba: Administración y Contaduría, Antropología, Arte, Bibliotecología y Ciencia de la Información, Ciencias de la Comunicación, Demografía, Derecho, Economía, Educación, Filosofía, Historia, Lingüística y Literatura, Política, Psicología, Relaciones Internacionales, Religión, Sociología. PERIODICA en cambio contiene: Agrociencias, Arquitectura, Astronomía, Biología, Ciencias de la Atmósfera, Computación, Física, Geofísica, Geología, Geografía, Ingeniería, Matemáticas, Medicina, Química, Oceanografía y Veterinaria

CLASE y PERIODICA ofrecen la siguiente información de cada artículo: autor(es), institución de trabajo de los autores, descripción hemerográfica (título de la revista, título del artículo, año de publicación, volumen, número y páginas del documento), temas, palabras clave $-\mathrm{y}$ en el caso de PERIODICA keywords-, resumen y enlace a texto completo en el caso de las revistas digitales

Estas bases se actualizan diariamente y cada una aumenta en un promedio de 10,000 nuevos registros por año. Desde hace un año, se incluyen resúmenes en los registros; y, desde 2005, enlace a los textos completos de revistas en formato digital. La totalidad de las revistas impresas incluidas en las bases se conservan en la Hemeroteca Latinoamericana de la DGB y se ofrece el servicio de documentación a los usuarios que lo soliciten personalmente o vía correo electrónico. Su consulta es gratuita a través de http://www.dgb.unam.mx

\subsubsection{IRESIE: Base de datos sobre educación iberoamericana}

Esta base de datos especializada en educación iberoamericana inició en 1979 en el otrora Centro Latinoamericano de Tecnología Educacional para la Salud. Actualmente es desarrollada en el Instituto de Investigaciones sobre la Universidad y la Educación, (IISUE) antes Centro de Estudios sobre la Universidad (CESU-UNAM).

La base contiene más de 65,000 registros de artículos teóricos, de revisión, reportes de investigación, experiencias docentes, ensayos, ponencias, conferencias y entrevistas publicadas en alrededor de 780 títulos de revistas en español y portugués que proceden de México, América Latina, El Caribe, España y Portugal. Las revistas se localizan en un $80 \%$ en la biblioteca del IISUE y el $20 \%$ restante en siete bibliotecas de las más importantes instituciones educativas de la ciudad de México.

Abarca toda la gama de aspectos relacionados con la educación, currículo, evaluación, didácticas especiales, filosofía de la educación, sociología de la educación, formación de profesores, etc., de todos los niveles y modalidades educativas.

Cada registro contiene: autor(es), descripción hemerográfica (título de la revista, título del artículo, año de publicación, volumen, número y páginas del documento), temas, resumen, biblioteca en la que se encuentra, y enlaces al texto completo en el caso de las revistas electrónicas

Esta base de datos se actualiza mensualmente y ofrece el servicio de documentación para la obtención de los artículos que se encuentran sólo en formato impreso. El acceso es gratuito en la dirección: www.iisue.unam.mx/iresie. 


\subsubsection{INFOBILA Información Bibliotecológica Latinoamericana}

Inició en 1985 como un proyecto de la biblioteca del Centro Universitario de Investigaciones Bibliotecológicas (CUIB) para concentrar y sistematizar la literatura especializada en bibliotecología, información y archivonomía producida en y sobre América Latina. A partir de 1995 se consolidó como proyecto regional de cooperación y desde entonces participan, además de México, varios países latinoamericanos entre ellos: Argentina, Bolivia, Brasil, Colombia, Costa Rica, Chile, Cuba, Puerto Rico, Perú.

Contiene más de 17,000 registros de referencias y resúmenes de artículos de revistas, capítulos de libros, ponencias, monografías y tesis, en algunos casos ofrece la liga a los textos completos, que sólo están accesibles para las instituciones integrantes del proyecto. Se puede consultar gratuitamente en: http://infocuib.laborales.unam.mx/ ibt/infoweb.ht $\mathrm{ml}$

\subsubsection{FILOS: Base de datos sobre Filosofía en México}

Base producida en el Instituto de Investigaciones Filosóficas de la UNAM. Recopila y sistematiza documentos sobre Filosofía de México.

Se inició en 1989. Contiene alrededor de 39,000 registros bibliográficos referenciales de libros, tesis, artículos de revistas, reseñas, bibliografías, obras de consulta, etcétera, publicados desde 1950 en México, la mayoría de los cuales se encuentran disponibles en la biblioteca del Instituto.

Los temas principales de la base de datos son: introducción a la filosofía, métodos filosóficos, didáctica de la filosofía, teoría de la filosofía, metafísica, ontología, axiología, filosofía de la religión, lógica, antropología filosófica, teoría del conocimiento, epistemología, ética, estética, teoría del arte, filosofía social, de la historia, de la educación, política, del derecho, del lenguaje, de la mente, hermenéutica, historia de la filosofía -filosofía antigua, medieval, moderna, de los siglos XIX, XX Y XXI, filosofía oriental, prehispánica y novohispana-, filosofía de la ciencia - de las matemáticas, de las ciencias naturales, de las ciencias sociales, de la psicología, de la inteligencia artificial y de la tecnología. Su acceso es gratuito en la dirección: www.filoso ficas.unam.mx/ bib/filos.htm
3.2.5. LATINDEX: Sistema regional para revistas científicas de América Latina, España y Portugal

Su objetivo es dar visibilidad a las revistas de la región y contribuir al mejoramiento de su calidad editorial, para ello compila, procesa y difunde información bibliográfica sobre las revistas y series monográficas publicadas en la región Iberoamericana.

Se inició en el Departamento de Bibliografía Latinoamericana de la DGB, como respuesta a las recomendaciones del Primer taller de publicaciones científicas, celebrado en 1994 en Guadalajara, Jalisco, en el que se señaló la necesidad de crear un sistema de información propio para revistas científicas producidas en América Latina y el Caribe. Al presente, ha logrado integrar esfuerzos de los siguientes países: Argentina, Brasil, Bolivia, Colombia, Chile, Costa Rica, Cuba, Ecuador, España, Guatemala, México, Nicaragua, Panamá, Perú, Portugal, Puerto Rico, República Dominicana, Uruguay y Venezuela.

Ha generado una serie de subproductos:

- Directorio: Inició en 1997, ofrece datos bibliográficos de 16,697 títulos de revistas. Permite conocer su trayectoria académica, cobertura temática, organismo editor, responsables editoriales, domicilio, forma de distribución, precio, bases de datos en las cuales están indizadas.

- Catálogo: Se generó a partir de 2002. Incluye 3,167 títulos seleccionados en cada país de acuerdo con criterios de calidad editorial.

- Revistas Electrónicas: Subconjunto de 2,627 títulos de revistas que tienen enlaces a texto completo.

Se actualiza diariamente y su consulta es gratuita en www.latindex.org

\subsubsection{Catálogos automatizados}

En la DGB también se elaboran desde 1978 catálogos electrónicos globales, que registran los materiales bibliográficos existentes en las 142 bibliotecas, a saber:

- LIBRUNAM, catálogo electrónico que compila todos los títulos de libros, en diferentes idiomas y de procedencia mundial que existen en las 142 bibliotecas de la UNAM (más de 2 millones de títulos) y proporciona la clasificación y ubicación en las diferentes bibliotecas.

- SERIUNAM, que contiene 53,000 títulos de revistas y proporciona la ubicación de los acervos en las diferentes bibliotecas de la 
UNAM y de otras instituciones de educación superior de México.

- TESIUNAM integra las más de 400,000 tesis de los egresados de la UNAM y de algunas otras instituciones que han realizado convenios con la UNAM, de estas 53,940 son electrónicas y se tiene acceso al texto completo únicamente a través de la red UNAM.

Estos catálogos han facilitado enormemente a los usuarios la localización de libros, revistas y tesis dispersos en las diferentes bibliotecas, y a los bibliotecarios la clasificación y catalogación de los materiales. Su acceso es gratuito en la dirección www.dgb.unam.mx

\section{Conclusiones}

La UNAM, organismo público, sostenido con recursos gubernamentales ha sido la institución mexicana pionera en la utilización de tecnologías de la información y se ha preocupado por adquirir, desarrollar, conservar y poner al alcance de la población en general recursos bibliográficos de manera gratuita.

Si bien la UNAM invierte una cantidad considerable de recursos en la compra de información que se produce principalmente en los Estados Unidos y otros países desarrollados, la cual es necesaria para el avance de la ciencia, también se ha preocupado y ha dado la debida importancia a la recolección, integración y sistematización de la información producida en México y otros países iberoamericanos que por el idioma y cercanía histórica, nos permite aproximarnos a problemas, formas de percibir, pensar y sentir, y así consolidarnos como una comunidad de intereses y fines compartidos.

Por otro lado, la gran variedad de campos de estudio que abarcan las bases de datos desarrolladas en la UNAM, así como la enorme cantidad de registros bibliográficos que se cuentan por millones, si sumamos las bases de datos multidisciplinarias y especializadas cuyo alcance es iberoamericano, aunado a otras características, tales como cubrir revistas de calidad, tener una antigüedad considerable, estar actualizadas, ser consistentes y estar disponibles gratuitamente en medios electrónicos, facilitan la realización de estudios bibliométricos sobre la producción científica latinoamericana, aspecto de primordial importancia para nuestro autoconocimiento y para detectar fortalezas y carencias en el desarrollo de nuestra ciencia, pensamiento y tecnología.

Así mismo, al contar con bases multidisciplinarias como CLASE y PERIODICA, se facilita la búsqueda más amplia de documentos referidos a un objeto de estudio, ya que debido a la tendencia actual de abordar los problemas desde múltiples enfoques, se requiere de varias disciplinas para ampliar las explicaciones y las bases especializadas en una disciplina no agotan los posibles abordajes contenidos en las diversas fuentes.

En contraste, la ventaja de consultar bases especializadas como IRESIE, FILOS o INFOBILA, consiste en la seguridad de encontrar con mayor precisión documentos sobre un aspecto específico del área, debido a la especificidad de sus documentos y también de los vocabularios controlados que se han desarrollado - por ejemplo, el Vocabulario Controlado del IRESIE y el Tesauro Latinoamericano en ciencia bibliotecológica y de la información, generado también en el CUIB.

Russell $(1998$, p. 176) señala la importancia de generar indicadores para medir la producción científica de América Latina utilizando bases de datos bibliográficas. En especial, se refiere al descuido que en este aspecto existe en las ciencias sociales y humanidades, y la tendencia de los científicos de las ciencias exactas y experimentales a publicar sus trabajos en revistas de países desarrollados.

Para concluir, la creación de bases de datos mexicanas e iberoamericanas ha contribuido también al control bibliográfico de la producción local, y regional, y ha permitido dar visibilidad a la ciencia generada en la región, además de propiciar el mejoramiento de la calidad editorial de las publicaciones y su distribución.

\section{Referencias}

CLASE, Citas Latinoamericanas en Ciencias Sociales y Humanidades (2008). México: UNAM. DGB, Departamento de Bibliografía Latinoamericana. http:// www. dgb.unam.mx (2008-09-06)

FILOS; base de datos sobre filosofía en México. (2008). México: UNAM, Instituto de Investigaciones Filosóficas. http://www.filosoficas.unam.mx/basefilos/ (2008-16-06).

INFOBILA: Información Bibliotecológica Latinoamericana (2008). México: UNAM, CUIB. http://infocuib.laborales. unam.mx/ ibt/infoweb.html (2008-05-05).

IRESIE: base de datos de educación iberoamericana (2008). México: UNAM; IISUE. www.iisue.unam.mx/iresie (200828-04).

LATINDEX: Sistema regional de información en línea para revistas científicas de América Latina, el Caribe, España y Portugal (2008). México: DGB. Departamento de Bibliografía Latinoamericana. www.latindex.unam.mx (2008-10-06)

PERIODICA, Indice de Revistas Latinoamericanas en Ciencias (2008). México: UNAM. DGB, Departamento de Bibliografía Latinoamericana. www.dgb.unam.mx (200809-06). 
Ranking mundial de Universidades en la WEB. Top Latino América. http://www.webometrics.info/top100_continent_ es.asp?cont=latin_america (2008-03-07).

Pérez Vitoria, Augusto (1982). La información en el marco de la cooperación científica internacional. // Inforum. 1 (1982) 1-31.

Russell, Jane M. (1998). El uso de las bases de datos bibliográficas en la definición de políticas en ciencia y tecnología en América Latina. // Centro Universitario de Investigaciones Bibliotecológicas. La Información en el inicio de la era electrónica, organización del conocimiento y sistemas de información. México: UNAM, CUIB, 1988. $1,172-185$

Universidad Nacional Autónoma de México. Dirección General de Planeación (2007). Agenda Estadística. http:// www.planeacion.unam.mx/agenda/2007/) (2008-05-07).

Universidad Nacional Autónoma de México. Dirección General de Bibliotecas (2007). Estadísticas del sistema bibliotecario 2007. (http://dgb.unam.mx/sbusite (2008-05-07). 